\title{
Hypophysitis induced by immune checkpoint inhibitors in a Scottish melanoma population
}

\author{
Khor Zhong Wei*,1 (iD), Mark Baxter ${ }^{1,2}$ \& Richard Casasola ${ }^{1}$ \\ ${ }^{1}$ Department of Oncology, Ninewells Hospital, Dundee, UK \\ ${ }^{2}$ University of Dundee, Dundee, UK \\ *Author for correspondence: Zhong.Khor@nhs.net
}

\begin{abstract}
Aim: This study aims to determine the incidence of all immune-mediated adverse events (IMAEs) with a focus on hypophysitis in patients with metastatic melanoma receiving immune checkpoint inhibitors (ICI). Methods: 51 patients with metastatic melanoma who received immune checkpoint inhibitors (ipilimumab, pembrolizumab and nivolumab) in Ninewells Hospital, Dundee between 2014 and 2018 were identified. Patient demographic data and outcomes were recorded retrospectively. Results: A total of 6 patients $(11.7 \%)$ developed hypophysitis, while 15 patients $(29.4 \%)$ developed IMAEs. A significant improvement in overall survival $(p=0.03)$ and progression-free survival $(p=0.041)$ was seen in patients who developed IMAEs compared with those who did not. Conclusion: This study demonstrates a high rate of hypophysitis in melanoma patients receiving ipilimumab. Careful monitoring of symptoms is crucial to detect and appropriately manage IMAEs.
\end{abstract}

First draft submitted: 25 November 2018; Accepted for publication: 19 March 2019; Published online: 18 April 2019

Keywords: adverse effects • hypophysitis • immunotherapy • ipilimumab • melanoma • nivolumab • outcome • pembrolizumab

To prevent recognition and destruction by cytotoxic $\mathrm{T}$ cells, cancer cells express ligands that activate T-cell inhibitory receptors, which downregulate their function [1,2]. Antibodies, which target these ligands and expose cancer cells to T-cell recognition, are known as immune checkpoint inhibitors (ICIs) and these agents are now the cornerstone of treatment for metastatic melanoma [3,4]. Among the immune inhibitory pathways that have been identified, the CTLA-4 and PD-1 pathways have demonstrated significant promise in the clinical setting.

Anti-CTLA-4 antibodies such as ipilimumab and anti-PD-1 antibodies such as pembrolizumab and nivolumab have been approved by the Scottish Medicine Consortium for the treatment of metastatic melanoma since 2014 [5,6]. An ever-growing evidence base demonstrates improved survival and patient outcomes with immunotherapy compared with traditional chemotherapy $[7,8]$. The majority of adverse events associated with ICIs are mild in nature; however, a proportion of patients do experience grade 3 and 4 toxicities [9-11]. Rarely, potentially fatal immunemediated adverse events (IMAEs) such as immune hypophysitis have been observed in patients receiving ICIs [12,13].

Hypophysitis is the inflammation of the pituitary gland, which often leads to irreversible hypopituitarism and can potentially lead to adrenal crisis if left untreated [14,15]. Clinical trial data have suggested rates of hypophysitis up to $17 \%$ in patients receiving ipilimumab $[16,17]$. In comparison, anti-PD 1 inhibitors such as pembrolizumab and nivolumab have demonstrated lower rates of hypophysitis $-0.47 \%$ based on a meta-analysis of nine randomized trials [18].

The reason underlying high rates of ipilimumab-mediated hypophysitis is thought to relate to expression of CTLA-4 in the pituitary. Experimental murine studies have demonstrated the expression of CTLA-4 coding RNA and elevated protein levels in pituitary cells. Analysis of a cohort of 20 patients demonstrated that pituitary antibodies, negative at baseline, developed in the seven patients with hypophysitis but not in the 13 patients without, suggesting a potential mechanism for immune-mediated hypophysitis through the development of anti-CTLA4 antibodies causing hypophysitis [19].

In our center, we have noticed a disproportionate increase in the incidence of hypophysitis in our cohort since the inception of these immunotherapeutic agents. Therefore, this study aims to investigate the incidence of all

Future $\because$ Medicine 
IMAEs with a focus on hypophysitis for patients with metastatic melanoma who received an ICI (ipilimumab, nivolumab and pembrolizumab) in Ninewells Hospital, Dundee, and to compare with published literature.

\section{Methods}

All patients with metastatic melanoma who received an ICI (ipilimumab, nivolumab and pembrolizumab) between January 2014 and August 2018 at our center (Ninewells Hospital, Dundee, Scotland) were identified from electronic chemotherapy records. Baseline patient demographic data were collected including age, sex, date of diagnosis, performance status on commencing treatment and treatment received.

In our center, we have two generic databases that store patient's medical notes and correspondence (Clinical Portal) as well as Laboratory investigations Integrated Clinical Environment (ICE). Furthermore, the database (Wisdom) contains all day to day progress for patients when they attend radiotherapy, chemotherapy or immunotherapy sessions. The practice nurse/doctor will document the procedure undertaken as well as monitor for any adverse reactions that the patient might have suffered from. The second database (Chemocare) records specifically the type, date and time of administration of immunotherapy, use of adjunct medication as well as any further reports of adverse reaction or ill health.

All four of these databases have been studied and every incidence of possible IMAEs including hypophysitis that had been reported and documented in these records has been included in this study. If present, management and outcome were recorded.

All patients who received ICIs at our center have had a toxicity assessment prior to every cycle, where baseline bloods inclusive of full blood count, urea and electrolytes, liver function test, bone profile, cortisol and thyroid function test were tested. Where there was a clinical indication, a more comprehensive hormone panel was carried out.

In this study, the diagnosis of hypophysitis was made based on the correlation of patient symptoms with the timing of administration of immunotherapy, as well as biochemical and radiological evidence demonstrating the development of hypopituitarism. A thyrotroph deficiency was defined as a low plasma T4 level $(<9.8$ pmol/l) with low or inappropriately normal levels of thyroid-stimulating hormone. Corticotroph deficiency was defined as low cortisol levels $(<275 \mathrm{nmol} / \mathrm{l})$ with low or inappropriately normal levels of adrenocorticotropic hormone. Gonadotroph deficiency was defined as low sex steroids (testosterone/estradiol) with inappropriately normal levels of follicle-stimulating hormone and luteinizing hormone levels. Last, lactotroph deficiency is defined as low levels of prolactin $(<6 \mathrm{mU} / \mathrm{l})$. Levels of growth hormone and IGF-1 were also recorded.

Recovery from hypophysitis was defined as a normalization of hormone profile as evidenced by blood tests as well as clinical recovery. Clinical recovery was defined as the patient no longer requiring hormone replacement therapy at the end of the follow-up period.

After collection of data, the study population was stratified into two groups according to treatment type and presence of IMAEs. Progression-free survival was defined as the time from commencing treatment to radiological evidence of progression. Overall survival was defined as the date from treatment commencement to date of death or end of the follow-up period. Grading of adverse events is based on the Common Terminology Criteria for Adverse Events version 5.0 [20].

All data were analyzed using SPSS (IBM SPSS Statistics for Windows, Version 21.0. Armonk, NY: IBM Corp.). Outcome data from this study were compared using Log-rank analysis to evaluate overall and progression-free survivals between both groups (where $\mathrm{p}<0.05$ is considered to be statistically significant). Local ethical approval was obtained prior to collection of data.

\section{Results}

Patient demographics

A total of 51 patients were identified as having received an ICI during the time period specified and were included in this study. Table 1 summarizes the characteristics of the study population. The majority of patients were male (74.5\%), had a performance status of $0-1(96 \%)$ and had a wild-type BRAF status (70.6\%). Additionally, 84.3\% of our patients have received surgical excision of their primary cutaneous melanoma previously prior to a relapse of their disease, which necessitate the use of immunotherapy. Most patients received no previous systemic treatments (86.3\%). Out of the seven patients who received previous systemic treatments, three had received ipilimumab immunotherapy previously, while the remaining four received conventional chemotherapy. To note, it is this 
Table 1. Summary of patient demographics.

\begin{tabular}{|c|c|c|}
\hline Demographic & & Number \\
\hline Age, median (months) & & $67 \pm 14.3$ \\
\hline Duration of follow-up, median (months) & & $7(1-55)$ \\
\hline Gender: & Male & $38(74.5 \%)$ \\
\hline & Female & $13(25.5 \%)$ \\
\hline Performance status: & 0 & $30(58.8 \%)$ \\
\hline & 1 & $19(37.2 \%)$ \\
\hline Cancer type: & Cutaneous melanoma & $46(90.2 \%)$ \\
\hline & Ocular melanoma & $5(9.8 \%)$ \\
\hline Primary cancer resected prior to therapy: & Yes & $43(84.3 \%)$ \\
\hline & No & $8(15.7 \%)$ \\
\hline Number of systemic treatments prior to therapy: & 0 & $44(86.3 \%)$ \\
\hline Nature of prior treatment: & Immunotherapy & 3 \\
\hline & Chemotherapy & 4 \\
\hline Type of immunotherapy: & Ipilimumab & $16(31.4 \%)$ \\
\hline & Nivolumab & $2(3.9 \%)$ \\
\hline & Pembrolizumab & $33(64.7 \%)$ \\
\hline Mean number of cycles of immunotherapy: & Ipilimumab & $3.5(2-4)$ \\
\hline & Nivolumab & $3.5(3-4)$ \\
\hline & Pembrolizumab & $14.7(1-50)$ \\
\hline Presence of hypophysitis: & Yes & $6(11.7 \%)$ \\
\hline & No & $45(88.3 \%)$ \\
\hline
\end{tabular}

center's policy to utilize immunotherapy as first line to treat metastatic melanoma, while $B R A F$ inhibitors such as dabrafenib are used second line where $B R A F$ mutation is positive.

All patients received their care in Ninewells Cancer Center, Dundee, from initiation of treatment until either the end point of the study or death, median follow-up was 7 months, ranging from 1 to 55 months. Two patients emigrated from the country for end of life care and were lost to follow-up.

\section{Hypophysitis characteristics \& management}

A total of six patients (11.7\%) developed clinically significant hypophysitis during their treatment (Table 2). Five of these patients had received ipilimumab (incidence of $31.3 \%$ in those who received ipilimumab) and one had received pembrolizumab (incidence of $3.0 \%$ in those receiving pembrolizumab). Table 2 shows the presenting features of each patient with hypophysitis.

Out of the six cases of hypophysitis, four patients required hospitalization, one patient (grade 2) was diagnosed and treated medically as an outpatient, while one patient had grade 1 hypophysitis and was completely asymptomatic (Patient 5 ). He was only diagnosed as routine blood monitoring has demonstrated mild corticotropin and thyrotropin deficiency.

The patients who developed hypophysitis presented with nonspecific symptoms such as headache, nausea and vomiting. Two patients developed other IMAEs, both of which had a grade 1 rash. Four out of six $(66.7 \%)$ patients were treated with systemic steroid therapy (Dexamethasone) and steroid treatment duration ranged from 7 to 39 days. Treatment dose of dexamethasone was tailored according to the clinical picture. The remaining two patients were not treated with dexamethasone as one of them was asymptomatic with only mild hormonal deficiencies (Patient 5), while the other (Patient 6) was started on intravenous hydrocortisone upon admission as per advice from the endocrinology team. 
Table 2. Cases of immunotherapy-induced hypophysitis.

\begin{tabular}{|c|c|c|c|c|c|c|}
\hline Case number & 1 & 2 & 3 & 4 & 5 & 6 \\
\hline Age & 70 & 70 & 64 & 77 & 46 & 24 \\
\hline Gender (M/F) & M & $\mathrm{F}$ & M & M & M & $\mathrm{F}$ \\
\hline Immunotherapy & Ipilimumab & Ipilimumab & Ipilimumab & Ipilimumab & Ipilimumab & Pembrolizumab \\
\hline $\begin{array}{l}\text { Treatment dose } \\
(\mathrm{mg} / \mathrm{kg})\end{array}$ & 3 & 3 & 3 & 3 & 3 & 2 \\
\hline $\begin{array}{l}\text { Number of cycles } \\
\text { prior to event }\end{array}$ & 3 & 4 & 4 & 2 & 3 & 35 \\
\hline $\begin{array}{l}\text { Hormonal defect } \\
\text { (time of diagnosis) }\end{array}$ & $\begin{array}{l}\text { Corticotroph, } \\
\text { thyrotroph, } \\
\text { gonadotroph }\end{array}$ & $\begin{array}{l}\text { Corticotroph, } \\
\text { thyrotroph }\end{array}$ & $\begin{array}{l}\text { Corticotroph, } \\
\text { gonadotroph }\end{array}$ & $\begin{array}{l}\text { Corticotroph, } \\
\text { thyrotroph, } \\
\text { gonadotroph }\end{array}$ & $\begin{array}{l}\text { Corticotroph, } \\
\text { thyrotroph }\end{array}$ & $\begin{array}{l}\text { Corticotroph, primary } \\
\text { hypothyroidism }\end{array}$ \\
\hline MRI findings & No abnormalities & No abnormalities & Not performed & No abnormalities & Not performed & Not performed \\
\hline Symptoms & Headache, syncope & Nausea & Tiredness & Headache & Asymptomatic & Nausea, vomiting \\
\hline $\begin{array}{l}\text { Dexamethasone dose } \\
\text { (mg) }\end{array}$ & 16 & 8 & 8 & 10 & Not given & Not given \\
\hline Recovery from event & No & No & Yes & No & Yes & No \\
\hline $\begin{array}{l}\text { Hormone } \\
\text { replacement (at the } \\
\text { end of follow-up) }\end{array}$ & $\begin{array}{l}\text { Levothyroxine, } \\
\text { testosterone, } \\
\text { hydrocortisone }\end{array}$ & $\begin{array}{l}\text { Hydrocortisone, } \\
\text { levothyroxine }\end{array}$ & None & $\begin{array}{l}\text { Hydrocortisone, } \\
\text { levothyroxine }\end{array}$ & None & $\begin{array}{l}\text { Hydrocortisone, } \\
\text { levothyroxine }\end{array}$ \\
\hline Additional IMAEs & None & Rash & None & Rash & None & None \\
\hline
\end{tabular}

\begin{tabular}{|c|c|c|c|}
\hline Case & Immunotherapy & Immune-mediated adverse effect & Grade \\
\hline 1 & Pembrolizumab & Dermatitis in lips & 1 \\
\hline 2 & Pembrolizumab & Rash & 1 \\
\hline 3 & Pembrolizumab & Rash & 1 \\
\hline 4 & Pembrolizumab & Hypothyroidism & 1 \\
\hline 5 & Pembrolizumab & Hypothyroidism & 2 \\
\hline 6 & Pembrolizumab & Hypothyroidism & 2 \\
\hline 7 & Pembrolizumab & Thrombocytopenia & 3 \\
\hline 8 & Pembrolizumab & Hypophysitis & 3 \\
\hline 9 & Ipilimumab & Hypophysitis & 1 \\
\hline 10 & Ipilimumab & Rash & 1 \\
\hline 11 & Ipilimumab & Colitis & 2 \\
\hline 12 & Ipilimumab & Hypophysitis & 2 \\
\hline 13 & Ipilimumab & Hypophysitis & 3 \\
\hline 14 & Ipilimumab & Hypophysitis & 3 \\
\hline 15 & Ipilimumab & Hypophysitis & 3 \\
\hline
\end{tabular}

All patients demonstrated corticotroph deficiency, four patients required lifelong hydrocortisone replacement while two patients recovered from their hypopituitarism at the end of the follow-up period. Thyroid function derangement was observed in five patients. Four patients demonstrated biochemical evidence of secondary hypothyroidism; however, one patient had concurrent primary hypothyroidism and hypophysitis. Four patients required lifelong levothyroxine replacement, while the remaining patients recovered. Gonadotrophic deficiency was observed in three patients on diagnosis; however, two patients recovered while one patient required testosterone replacement at the end of follow-up. MRI was performed shortly (within 1 week) after presentation in three patients, all of which demonstrated unremarkable findings.

Including the six patients who developed hypophysitis, a further nine (18\%) patients (Table 3) developed IMAEs, most of which were grade 1-2; three developed primary hypothyroidism, with two requiring levothyroxine replacement. There were six cutaneous reactions (rash and dermatitis) and one incident of grade 3 immune-mediated thrombocytopenia and colitis each. 


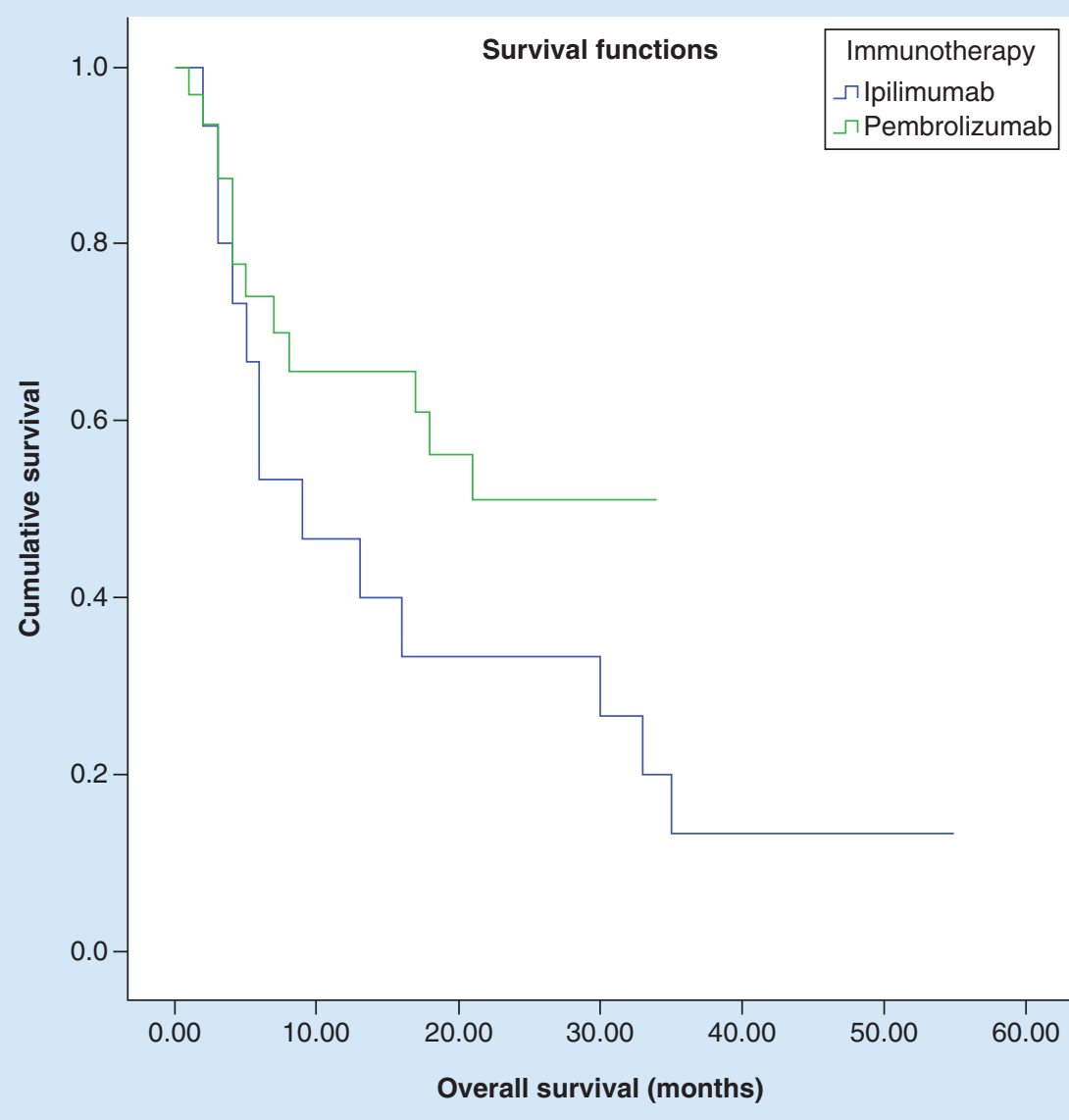

Figure 1. Kaplan-Meier survival curve comparing overall survival of patients who received ipilimumab versus those who received pembrolizumab $(p=0.180)$.

\section{Table 4. Characteristics of patients treated with ipilimumab versus pembrolizumab.}

\begin{tabular}{|llll}
\hline Patient Characteristics & Ipilimumab $(\mathbf{n = 1 6 )}$ & Pembrolizumab $(\mathbf{n = 3 3 )}$ & $\mathbf{p}$-value \\
\hline Age & 62.63 & 64.33 & 0.691 \\
\hline Gender (\% male) & $68.75 \%$ & $75.76 \%$ & 0.624 \\
\hline Overall survival (median) & 18.3 months; & 21.6 months; & 0.180 \\
& $95 \%$ Cl: $9.2-27.4$ & $95 \%$ Cl: $16.4-26.7$ & 19.8 months; \\
\hline Progression-free survival (median) & 12.1 months; & $95 \%$ Cl: $13.9-25.7$ & 0.042 \\
\hline
\end{tabular}

\section{Patient outcome data}

There was no statistically significant difference in overall survival (Figure $1 ; 21.6$ vs 18.3 months; $\mathrm{p}=0.180$ ) between patients who received pembrolizumab and those who received ipilimumab (Table 4). However, pembrolizumab provides a better progression-free survival for patients as compared with ipilimumab (Figure 2; 19.8 vs 12.1 months; $\mathrm{p}=0.042)$.

Log-rank (Mantel Cox) analysis (Figures $3 \& 4$ ) demonstrated a significant difference in overall survival (20.6 vs 11.1 months, $\mathrm{p}=0.030)$ and progression-free survival ( 16.7 vs 6.38 months; $\mathrm{p}=0.041)$ among patients who develop IMAEs (Table 5). There is no observable difference in age or gender distribution seen in both groups. At the end point of follow-up, only two patients (33.33\%) recovered their pituitary function and did not require lifelong hormone replacement. 


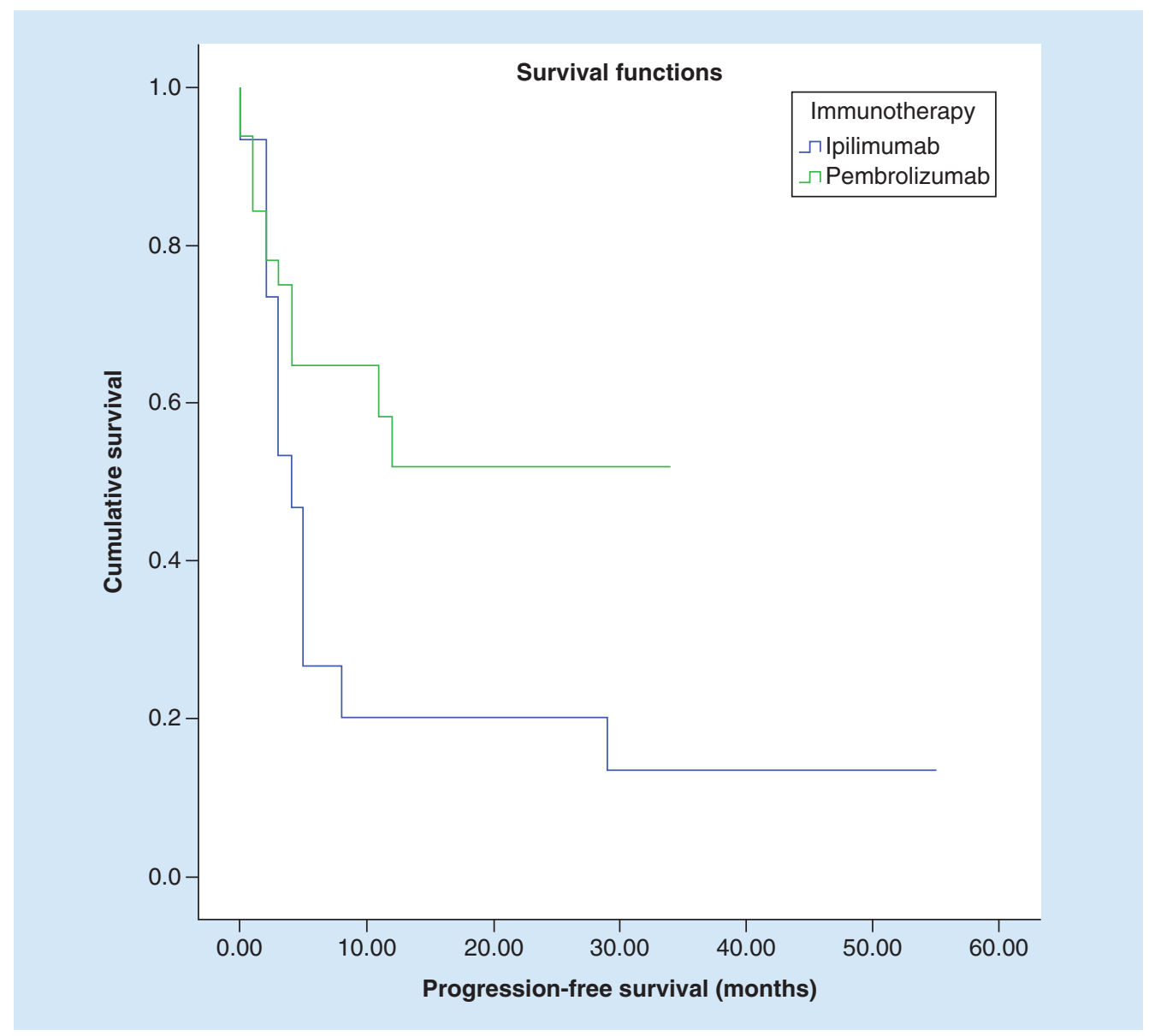

Figure 2. Kaplan-Meier survival curve comparing progression-free survival of patients who received ipilimumab versus those who received pembrolizumab $(p=0.042)$.

\begin{tabular}{|c|c|c|c|}
\hline Patient characteristics & Affected $(n=15)$ & Not affected $(n=36)$ & p-value \\
\hline Age & 64.5 & 63.6 & 0.846 \\
\hline Gender & $73.3 \%$ & $75.0 \%$ & 0.906 \\
\hline Overall survival (median) & $\begin{array}{l}20.6 \text { months; } \\
95 \% \text { Cl: } 10.9-30.3\end{array}$ & $\begin{array}{l}11.1 \text { months; } \\
95 \% \text { Cl: } 7.8-14.5\end{array}$ & 0.030 \\
\hline Progression-free survival (median) & $\begin{array}{l}16.7 \text { months; } \\
95 \% \mathrm{Cl}: 6.9-26.6\end{array}$ & $\begin{array}{l}6.38 \text { months; } \\
95 \% \text { Cl: 3.7-9.1 }\end{array}$ & 0.041 \\
\hline
\end{tabular}

\section{Discussion}

ICIs have revolutionized the treatment of metastatic melanoma. With growing experience and ongoing studies evaluating the efficacy and safety of agents such as ipilimumab and pembrolizumab, the initiation of such treatments can be confidently made. Increasing awareness of the potential side effects has also led to the development of adverse event treatment guidelines [21,22]. This study aimed to quantify objectively the adverse event profile of these agents in the melanoma patient population in the east of Scotland.

The use of ipilimumab to treat melanoma has been discontinued in this cancer center from 2015 due to growing concerns of its toxicity and with the introduction of newer and safer agents such as pembrolizumab and nivolumab. Combination treatment of ipilimumab and nivolumab is also avoided due to toxicity. This study has demonstrated that anti-PD1 inhibitors provide superior patient outcomes in terms of progression-free survival and safety. Multiple Phase III clinical trials have demonstrated improved overall and progression-free survivals for pembrolizumab compared with ipilimumab [23-26]. 


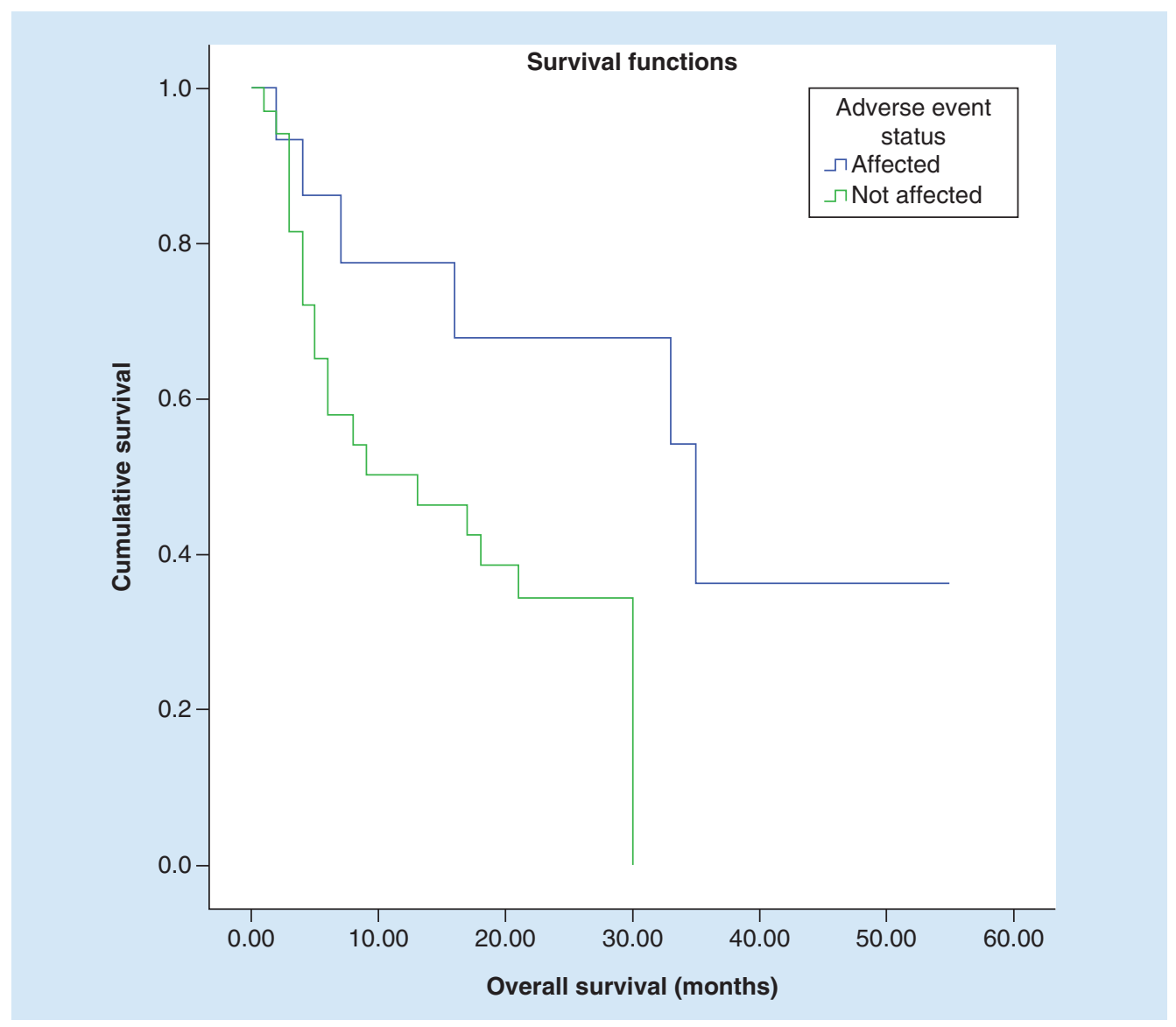

Figure 3. Kaplan-Meier survival curve comparing overall survival of patients affected by immune-mediated adverse reaction versus those who did not $(p=0.030)$.

In this population, patients taking pembrolizumab developed a lower rate of IMAEs compared with those taking ipilimumab. Moreover, these adverse reactions are often mild and self-limiting, with most patients having only grade 1 and 2 toxicity. This is in contrast to ipilimumab where $42.9 \%$ of patients suffered from grade 3 IMAE in contrast to $25 \%$ in the pembrolizumab cohort. Three patients $(9 \%)$ developed hypothyroidism in the pembrolizumab cohort, consistent with the findings of a large systematic review, which found that hypothyroidism is the most common endocrine disorder seen, at a rate of $8 \%[27]$.

All six patients who developed hypophysitis demonstrated a variety of hormonal deficiencies, albeit of varying severity. For example, one patient was asymptomatic and made a complete recovery of his pituitary function without need for systemic steroid treatment or hormone replacement. In contrast, four patients required hospitalization, high-dose dexamethasone or intravenous hydrocortisone replacement to effectively manage their symptoms. Ultimately, the majority of these patients required long-term hormone replacement. This observation is consistent with the often unpredictable natural history and severity of hypophysitis that has been described in the current literature [28,29]. This further elucidates the necessity of tailoring the treatment according to the individual patient, with appropriate monitoring to help determine whether to continue with the immunotherapy regime or to switch to other conventional therapies [30,31].

Another interesting finding in this study is the lack of radiological findings in the patient cohort; three out of six patients (50\%) who developed hypophysitis received a brain MRI, which failed to demonstrate any significant abnormalities. This is in contrast to the typical findings of an enlarged pituitary gland or infundibulum that is commonly described in other studies investigating this [32-34]. However, while MRI imaging can aid in reaching the diagnosis, it lacks in specificity particularly when distinguishing hypophysitis and pituitary adenomas or incidentalomas. As such, careful correlation with clinical history and biochemical findings is still necessary [14]. 


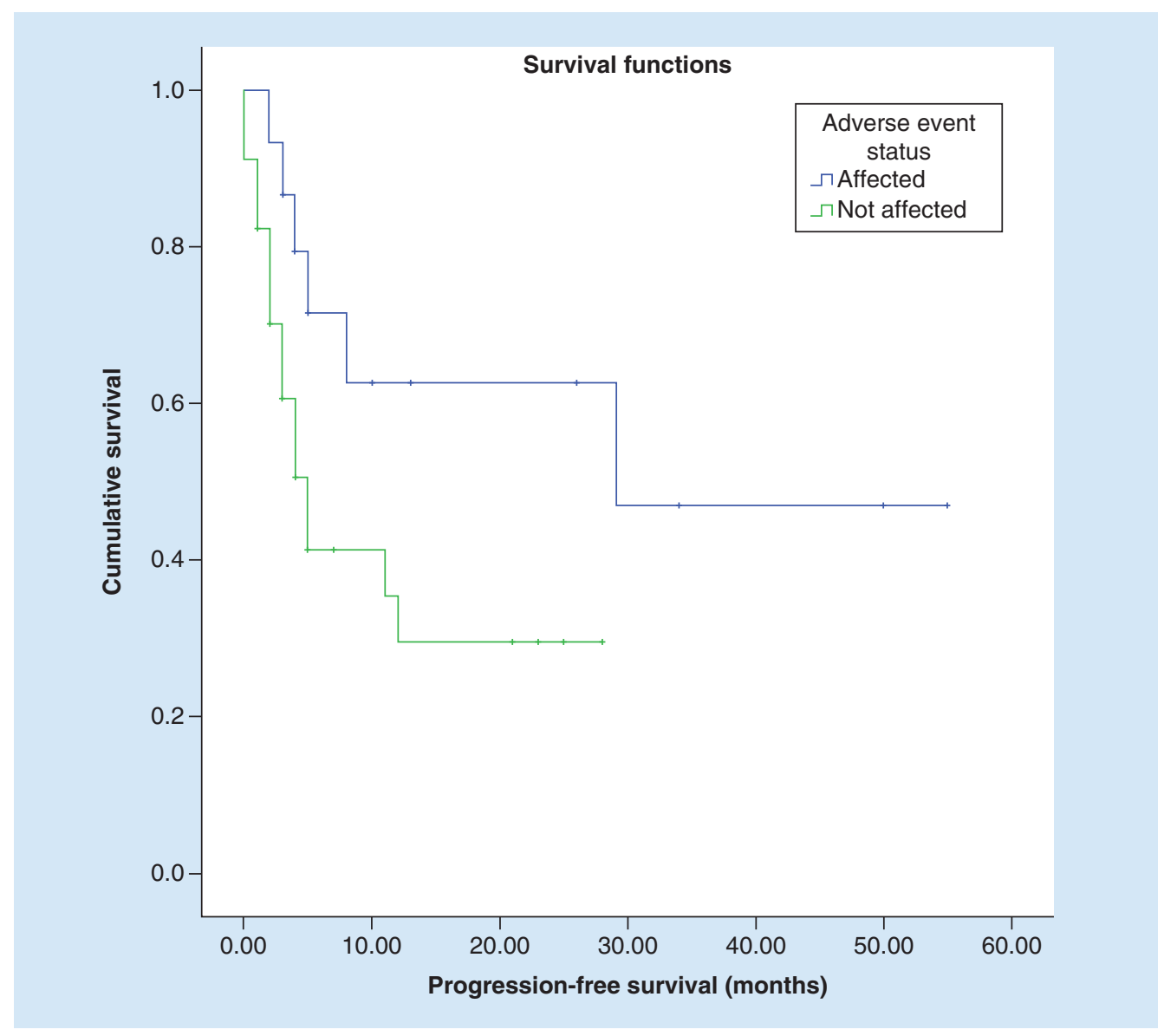

Figure 4. Kaplan-Meier curve comparing progression-free survival of patients affected by immune-mediated adverse reaction versus those who did not $(p=0.041)$.

Furthermore, this study population has a lower percentage of $B R A F$ mutant melanoma (11.8\%) compared with what has been reported in the literature, where a rate of approximately $50 \%$ is expected [35]. This finding may represent the variation in the local population leading to a mismatch with other trails population or could be coincidental due to the study's small sample size.

With a small sample size $(\mathrm{n}=51)$, it is difficult to draw inferences on the prevalence of hypophysitis from this cohort. However, the study data seem to suggest higher rates of hypophysitis both among patients taking ipilimumab and pembrolizumab. The current literature suggests an incidence of $0-17 \%$ for hypophysitis among the ipilimumab patient cohort [17]. To note, while a hypophysitis rate of $0.47 \%$ has been quoted in the meta-analysis performed by Costa $e$ al. assessing the toxicity profile of pembrolizumab and nivolumab in solid tumors, Wang et $a l$. have demonstrated in their study that pembrolizumab causes a hypophysitis rate of $<0.01 \%$ specifically in the metastatic melanoma patient cohort [18,27]. To compare, this study's cohort demonstrates a rate of 31.3 and $3.0 \%$ for ipilimumab and pembrolizumab, respectively.

A possible explanation of the higher rates seen is that there has been an increased vigilance in monitoring patients for IMAEs and a lower threshold to investigate patients presenting with nonspecific symptoms in recent years. Indeed, many earlier studies have attributed a possibility of under-reporting IMAEs due to poor recognition [36,37].

The overall incidence for all grade IMAEs for this cohort is 43.8 and $27.3 \%$ for ipilimumab and pembrolizumab, respectively. To compare, two meta-analysis analyzing the adverse effect profile of these two immunotherapy agents reported an incidence of 61 and $26.8 \%$ for ipilimumab and pembrolizumab, respectively [17,27]. Our study data broadly match the incidence of reported IMAEs in clinical trials - although we do recognize that our rates for ipilimumab are lower. This observation is likely associated to a smaller ipilimumab patient cohort due to the shorter duration of use of this agent in our center before it was discontinued, allowing less IMAEs to be recorded. 
At the time of treatment, ipilimumab and pembrolizumab were prescribed at a dose of 3 and $2 \mathrm{mg} / \mathrm{kg}$, respectively. We are aware that flat dosing is now standard of care and this is under review at our center. However, this study was not able to elicit a demonstrable difference in cumulative dose between patients who develop hypophysitis and those who did not, as has been shown in other studies $[17,27,35]$. This is perhaps again attributed to the small sample size.

Interestingly, the data from this study demonstrated statistically and clinically significant improvement in overall and progression-free survivals for patients taking ICIs who develop IMAEs. Though the exact mechanism for this is unknown, multiple studies conducted have also similarly reported improved tumor response rate and increased survival rates with patient who develop IMAEs [38-40].

\section{Conclusion}

This study demonstrates that the incidence of IMAEs and, in particular, hypophysitis in this center's population is higher in patients on ipilimumab compared with pembrolizumab. There is an observable increase in incidence of hypophysitis in this cohort as compared with the general study population. The data also support emerging evidence that suggests better overall and progression-free survivals in patients who develop immune-mediated adverse reactions compared with those who did not. With the increased use on ICIs, medical professionals need to be aware of the potential serious adverse events and need to become better at recognizing and managing these specific toxicities.

\section{Future perspective}

From this author's perspective, there are many new emerging immunotherapies that are currently being trialled at different phases. New immunotherapies and new combinations of immunotherapies/targeted therapy/chemotherapy are now being actively investigated; these will provide clinicians with far more options for treatment as compared with what we currently have now. However, as these treatments are introduced into clinical practice, great care has to be taken to avoid increasing morbidity and mortality in the pursuit of a cure. With the introduction of new treatment regimens and agents, new protocols for monitoring and guidance for managing associated adverse events will be needed to ensure that patients obtain maximal benefit from these treatments. Another area of great improvement that can be anticipated is the development of biomarkers, which will allow us to personalize treatment for our patients where laboratory tests will help us select patients who will respond to certain treatment or help us exclude patients who might develop severe adverse reactions.

\section{Summary points}

- Immune checkpoint inhibitors are effective in treating metastatic melanoma, but are known to cause immune-mediated adverse events (IMAEs), which can result in lifelong endocrine complications.

- Out of 51 patients with metastatic melanoma treated with immune checkpoint inhibitors in Ninewells Hospital, Dundee, a total of 6 patients (11.7\%) developed clinically significant hypophysitis, 5 patients had received ipilimumab and 1 had received pembrolizumab.

- A further nine patients developed other IMAEs; three developed primary hypothyroidism, with a further six cutaneous reactions and one incident of immune-mediated thrombocytopenia and colitis each.

- There is a greater rate of the development of hypophysitis in our center than reported in published literature.

- A significant difference in overall survival (20.6 vs 11.1 months, $p=0.03$ ) and progression-free survival (16.7 vs 6.4 months, $p=0.041$ ) was observed among patients who develop IMAEs compared with those who did not.

\section{Author contributions}

The paper is the result of joint research, the contribution of every author is comparable to the others.

\section{Financial \& competing interests disclosure}

The authors have no relevant affiliations or financial involvement with any organization or entity with a financial interest in or financial conflict with the subject matter or materials discussed in the manuscript. This includes employment, consultancies, honoraria, stock ownership or options, expert testimony, grants or patents received or pending, or royalties.

No writing assistance was utilized in the production of this manuscript. 


\section{Ethical conduct of research}

The authors state that they have obtained appropriate Institutional Review Board approval or have followed the principles outlined in the Declaration of Helsinki for all human or animal experimental investigations. In addition, for investigations involving human subjects, informed consent has been obtained from the participants involved. No formal ethics review committee needed as no human patient contact was needed. Approval from clinical lead/line manager, clinical supervisor and lead pharmacist obtained prior to starting the project as per local unit policy.

\section{Open access}

This work is licensed under the Attribution-NonCommercial-NoDerivatives 4.0 Unported License. To view a copy of this license, visit http://creativecommons.org/licenses/by-nc-nd/4.0/

\section{References}

Papers of special note have been highlighted as: • of interest; $\bullet \bullet$ of considerable interest.

1. Pardoll DM. The blockade of immune checkpoints in cancer immunotherapy. Nat. Rev. Cancer 12, 252-264 (2012).

2. Sharma P, Allison JP. The future of immune checkpoint therapy. Science 348, 56-61 (2015).

3. Ott PA, Hodi FS, Robert C. CTLA-4 and PD-1/PD-L1 blockade: new immunotherapeutic modalities with durable clinical benefit in melanoma patients. Clin. Cancer Res. 19, 5300-5309 (2013).

4. Munhoz RR, González AF, Reed VA, Postow MA. Targeting immune checkpoints in melanoma: an update. Melanoma Manag. 2(4), 339-352 (2015).

5. Scottish Medicine Consortium. Ipilumumab. (2018). www.scottishmedicines.org.uk/medicines-advice/ipilimumab-yervoy-abbreviatedsubmission-smc2094/

6. Scottish Medicine Consortium. Pembrolizumab. (2019). www.scottishmedicines.org.uk/medicines-advice/pembrolizumab-keytruda-fullsubmission-108615/

7. Callahan MK, Postow MA, Wolchok JD. Targeting T cell co-receptors for cancer therapy. Immunity 44, 1069-1078 (2016).

8. Karlsson AK, Saleh SN. Checkpoint inhibitors for malignant melanoma: a systematic review and meta-analysis. Clin. Cosmet. Investig. Dermatol. 10, 325-339 (2017).

9. Naidoo J, Page DB, Li BT et al. Toxicities of the anti-PD-1 and anti-PD-L1 immune checkpoint antibodies. Ann. Oncol. 26, 2375-2391 (2015).

10. Joshi MN, Whitelaw BC, Palomar MT, Wu Y, Carroll PV. Immune checkpoint inhibitor-related hypophysitis and endocrine dysfunction: clinical review. Clin. Endocrinol. 3, 331-339 (2016).

11. Myers G. Immune-related adverse events of immune checkpoint inhibitors: a brief review. Curr. Oncol. 25(5), 342-347 (2018).

12. Wang DY, Salem JE, Cohen JV et al. Fatal toxic effects associated with immune checkpoint inhibitors: a systematic review and meta-analysis. JAMA Oncol. 4(12), 1721-1728 (2018).

13. Varricchi G. Marone G, Mercurio V, Galdiero MR, Bonaduce D, Tocchetti CG. Immune checkpoint inhibitors and cardiac toxicity: an emerging issue. Curr. Med. Chem. 25, 1327-1339 (2018).

14. Caturegli P. Newschaffer C, Olivi A, Pomper MG, Burger PC, Rose NR. Autoimmune hypophysitis. Endocr. Rev. 26(5), 599-614 (2005).

15. Reimondo G, Bovio S, Allasino B et al. Secondary hypoadrenalism. Pituitary 11, 147-154 (2008).

16. Tarhini A. Immune-mediated adverse events associated with ipilimumab CTLA-4 blockade therapy: the underlying mechanisms and clinical management. Scientifica, 2013, 857519 (2013).

- Tarhini's study clearly elucidates the proposed mechanism and management of immune-mediated adverse events caused by ipilimumab.

17. Bertrand A, Kostine M, Barnetche T, Truchetet ME, Schaeverbeke T. Immune related adverse events associated with anti-CTLA-4 antibodies: systematic review and meta-analysis. BMC Med. 13, 211 (2015).

-. A meta-analysis that studies the prevalence of immune-mediated adverse events in patients taking ipilimumab. Provides valuable information with regards to other's study finding and allow meaningful comparison to be made.

18. Costa R, Carneiro BA, Agulnik M et al. Toxicity profile of approved anti-PD-1 monoclonal antibodies in solid tumors: a systematic review and meta-analysis of randomized clinical trials. Oncotarget 8(5), 8910-8920 (2017).

-. A meta-analysis that reports the prevalence of immune-mediated adverse events in patients taking anti-PD-1 inhibitors such as pembrolizumab and nivolumab. Provides valuable information with regards to other's study finding and allow meaningful comparison to be made.

19. Iwama S, De Remigis A, Callahan MK, Slovin SF, Wolchok JD, Caturegli P. Pituitary expression of CTLA-4 mediates hypophysitis secondary to administration of CTLA-4 blocking antibody. Sci. Transl. Med. 6(230), 230ra45 (2014). 
20. US Department of Health and Human Services. Common Terminology Criteria for Adverse Events (CTCAE) version 5.0. (2017). https://ctep.cancer.gov/protocoldevelopment/electronic_applications/docs/CTCAE_v5_Quick_Reference_5x7.pdf

21. Haanen JBAG, Carbonnel F, Robert C et al. Management of toxicities from immunotherapy: ESMO Clinical Practice Guidelines for diagnosis, treatment and follow-up. Ann. Oncol. 28(4), iv119-iv142 (2017).

22. Puzanov I, Diab A, Abdallah K et al. Managing toxicities associated with immune checkpoint inhibitors: consensus recommendations from the Society for Immunotherapy of Cancer (SITC) Toxicity Management Working Group. J. Immunother. Cancer 5(1), 95 (2017).

23. Schachter J, Ribas A, Long GV et al. Pembrolizumab versus ipilimumab for advanced melanoma: final overall survival results of a multicentre, randomised, open-label Phase III study (KEYNOTE-006). Lancet 390, 1853-1862 (2017).

- Schachter study is a Phase III clinical trial, which provides evidence of superior efficacy and safety of pembrolizumab over nivolumab.

24. Robert C, Schachter J, Long GV et al. Pembrolizumab versus ipilimumab in advanced melanoma. N. Eng. J. Med. 372, 2521-2532 (2015).

25. Petrella TM, Robert C, Richtig E et al. Patient-reported outcomes in KEYNOTE-006, a randomised study of pembrolizumab versus ipilimumab in patients with advanced melanoma. Eur. J. Cancer 86, 115 (2017).

26. Carlino MS, Long GV, Schadendorf D et al. Outcomes by line of therapy and programmed death ligand 1 expression in patients with advanced melanoma treated with pembrolizumab or ipilimumab in KEYNOTE-006: a randomised clinical trial. Eur. J. Cancer 101, 236-243 (2018).

27. Wang PF, Chen Y, Song S-Y et al. Immune-related adverse events associated with anti-PD-1/PD-L1 treatment for malignancies: a meta-analysis. Front. Pharmacol. 8, 730 (2017).

-• Another meta-analysis that studies immune-mediated adverse events associated with anti-PD-1 inhibitors. Provides valuable information with regards to other's study finding and allow meaningful comparison to be made.

28. Bellastella G, Maiorino ML, Bizzarro A et al. Revisitation of autoimmune hypophysitis: knowledge and uncertainties on pathophysiological and clinical aspects. Pituitary 19(6), 625-642 (2016).

29. Iglesias P. Cancer immunotherapy-induced endocrinopathies: clinical behavior and therapeutic approach. Eur. J. Intern. Med. 47, 6-13 (2018).

30. González-Rodríguez E, Rodríguez-Abreu D. Immune checkpoint inhibitors: review and management of endocrine adverse effects. Oncologist 21, 804-816 (2016).

31. Davies M, Duffield EA. Safety of checkpoint inhibitors for cancer treatment: strategies for patient monitoring and management of immune-mediated adverse events. Immunotargets Ther. 6, 51-71 (2017).

32. Faje AT, Sullivan R, Lawrence D et al. Ipilimumab-induced hypophysitis: a detailed longitudinal analysis in a large cohort of patients with metastatic melanoma. J. Clin. Endocrinol. Metab. 99(11), 4078-4085 (2014).

- A longitudinal study with good methodology, which follows up a cohort of patients who developed hypophysitis when on ipilimumab.

33. Carpenter KJ, Murtagh RD, Lilienfeld H, Weber J, Murtah FR. Ipilimumab-induced hypophysitis: MR imaging findings. AJNR Am. J. Neuroradiol. 30(9), 1751-1753 (2009).

34. Miller AH, Brock P, Yeung SCJ. Pituitary dysfunction. A case series of immune checkpoint inhibitor-related hypophysitis in an emergency department. Ann. Emerg. Med. 68, 249-250 (2016).

35. Ascierto PA, Kirkwood JM, Grob JJ et al. The role of BRAF V600 mutation in melanoma. J. Transl. Med. 10, 85-93 (2012).

36. Ibrahim R, Berman DM, DePril V et al. Ipilimumab safety profile: summary of findings from completed trials in advanced melanoma. $J$. Clin. Oncol. 29, abstract no. 8583 (2011).

37. Albarel F, Gaudy C, Castinetti F et al. Long-term follow-up of ipilimumab-induced hypophysitis, a common adverse event of the anti-CTLA-4 antibody in melanoma. Eur. J. Endocrinol. 1722, 195-204 (2015).

38. Downey SG, Klapper JA, Smith FO et al. Prognostic factors related to clinical response in patients with metastatic melanoma treated by CTL-associated antigen-4 blockade. Clin. Cancer Res. 13, 6681-6688 (2007).

39. Attia P, Phan GQ, Maker AV et al. Autoimmunity correlates with tumor regression in patients with metastatic melanoma treated with anti-cytotoxic T-lymphocyte antigen-4. J. Clin. Oncol. 23, 6043-6053 (2005).

- Provides some insight as to the phenomena of autoimmunity and positive treatment outcomes in immunotherapy and melanoma.

40. Bouwhuis MG, Ten Hagen TLM, Suciu S, Eggermont AM. Autoimmunity and treatment outcome in melanoma. Curr. Opin. Oncol. 23, 170-176 (2011). 
